\title{
Adaptive Active Power Sharing Techniques for DC and AC Voltage Control in a Hybrid DC/AC Microgrid
}

\author{
Ángel Navarro-Rodríguez, Pablo García, Ramy Georgious and Jorge García \\ Dept.of Electrical, Electronics, Systems \& Computers Engineering \\ University of Oviedo, LEMUR Group \\ Gijón, 33204, Spain \\ Email: navarroangel@uniovi.es, garciafpablo@uniovi.es, georgiousramy@uniovi.es, garciajorge@uniovi.es
}

\begin{abstract}
This paper deals with the AC and DC dynamic voltage control in a hybrid DC/AC Microgrid (MG) with central and distributed Battery Energy Storage Systems (BESSs), applying a power sharing mechanism between the different devices in the MG. The MG is composed by a multiport transformation center and two fixed frequency 3 phase AC Nanogrids (NGs) coupled to a DC bus through 3-phase Power Electronic Converters (PECs). The system pursues to minimize the dependence on the utility grid and the stress in the MGs central BESS, while increasing the power handling capability and the overall system stability during islanding condition. In order to approach the proposed aim, two main concerns are studied in this paper: an adaptive power sharing mechanism between the DC bus and the AC NGs for DC voltage control, and the design and implementation of an AC dynamic local voltage compensator based on Distributed Energy Storage System (DESS). The proposed techniques are validated through simulations and experimental results.
\end{abstract}

\section{INTRODUCTION}

The increasing concern about environmental issues and the rising popularity of concepts as local generation and selfconsumption have led to an increasing interest on alternatives to the conventional utility grid as Microgrids (MGs), Nanogrids (NGs) and Smart grids (SGs). Despite its advantages, the weakness and stability problems associated to MGs have been considered since its appearance, demanding significant research interests, specially regarding the power quality improvement [1], [2]. Furthermore, with the appearance of hybrid DC/AC MGs, where distributed energy resources (DERs) and loads may share/draw power in both the AC grid and the DC lines, new MG issues appears as the stability and grid quality in DC and AC [3], [4].

Several topologies for hybrid MGs have been proposed during the last years, classified by the interconnection with the mains and the connection between the DC and the AC

The present work has been partially supported by the predoctoral grants program Severo Ochoa for the formation in research and university teaching of Principado de Asturias PCTI-FICYT under the grant ID BP14-135. This work also was supported in part by the Research, Technological Development and Innovation Program Oriented to the Society Challenges of the Spanish Ministry of Economy and Competitiveness under grant ENE2016-77919-R and by the European Union through ERFD Structural Funds (FEDER). networks [5]. However, most of the examples in the literature are based on a direct connection with the mains in the $\mathrm{AC}$ grid and an interlinking converter between the DC and $\mathrm{AC}$ buses, being susceptible to contingencies in the mains [3], [4], [6], [7]. In contrast, the MG topology proposed in [8] and shown in Fig. 1, consist in a two-stage completely isolated topology with multiple AC Nanogrids (NGs) that allows to decouple the DC and AC buses from the utility grid through a MG head converter (MGHC). This reduces the impact of distributed generation (DG) in the mains and the sensitivity of the MG to contingencies in the utility grid, being the selected topology for this study. Moreover, the required modification to the already existing infrastructure is minimized, since there are no changes in the distribution system downstream of the transformation center.

Regarding the control system, several control schemes (central controller, master-slave, droop-based control, hybrid approaches ...) are found in the literature to ensure the voltage/frequency control and power sharing in $\mathrm{AC}$ distribution networks [6], [9]-[11], and DC grids [12], [13]. In the particular application of hybrid MGs, some control solutions have been proposed [14]. However, most of them are based on a $\mathrm{DC} / \mathrm{AC}$ interlinking converter operated in grid following mode [3], [4], [6], [7].

Conversely, the topology in [8] is based on DC/AC 3phase 2-level interlinking converters operated in grid forming mode (slack mode). Compared to droop control strategies, grid forming control methods based on master-slave or multislack grid control, with fixed frequency, can simplify the MG design with high presence of DERs interfaced by power electronic converters (PECs), and renewable sources operated under maximum power point tracking (MPPT) [12], [13], [15].

This tight regulation in the interlinking converters, hereinafter referred to as Nanogrid head converters (NGHCs) [8], allows for decoupling the AC from the DC bus dynamics, thus, the NGs behave as CPLs for the LVDC. Counter-intuitive, this might become an issue if the LVDC present a low inertia, for instance during islanding mode or under low DC bus capacitance. Therefore, the solution to avoid critical dynamic 